\title{
Integrals for IBS and Beam Cooling
}

\author{
A. Burov \\ Fermi National Accelerator Laboratory, USA
}

\begin{abstract}
Simulation of beam cooling usually requires performing certain integral transformations every time step or so, which is a significant burden on the CPU. Examples are the dispersion integrals (Hilbert transforms) in the stochastic cooling, wake fields and IBS integrals. An original method is suggested for fast and sufficiently accurate computation of the integrals. This method is applied for the dispersion integral. Some methodical aspects of the IBS analysis are discussed.
\end{abstract}

Keywords: electron cooling, stochastic cooling, intra-beam scattering

PACS: 29.20.-c, 29.27.-a

\section{INTRODUCTION}

Simulation of beam cooling usually requires intensity effects to be taken into account. For stochastic cooling, computation of the beam response requires Hilbert transformation of the distribution function being repeated many times for the simulation process. If the beam is intense enough, intra-beam scattering (IBS) and instabilities normally have to be taken into account both for stochastic and electron cooling scenarios.

From a computational point of view, intensity effects require integral transformations of the evolving distribution function to be repeated every time step or so, which puts a heavy burden on the CPU. The cooling scenario is characterized by many parameters. Some of these parameters have to be optimized, other have to be below some tolerances. One of the main purposes of the modeling is to find these optimal points and limits. Solving problems like that requires numerous cut-and-try runs of the modeling program for various points in a multi-dimensional space of parameters. That is why the program has to be sufficiently fast, taking not longer than a few minutes per a single run. To meet this goal, all the mentioned composites of the beam evolution have to be computed at a proper compromise between the duration and accuracy. This paper describes some ideas which the author found useful in his computations for electron cooling projects of antiprotons in the Recycler, FNAL [1] and heavy ions in RHIC, BNL [2].

\section{GUESS METHOD OF INTEGRAL TRANSFORMATION}

\section{General Description}

Imagine that you have to calculate an integral like

$$
\chi(u)=\int_{-\infty}^{\infty} f(v) K(u, v) d v
$$

where $f(v)$ is a distribution function, or its derivative, or something related to the distribution function, and $\boldsymbol{K}(\boldsymbol{u}, \boldsymbol{v})$ is a given kernel, which might have an integrable singularity, usually at $\boldsymbol{u}=\boldsymbol{v}$. Such integrals appear in stochastic cooling as the dispersion integrals (Hilbert transformations), and for intra-beam scattering as the friction force and diffusion coefficient. These singular integrals have to be calculated numerically at every point of the phase space (for every $\boldsymbol{u}$ ), and at every (or so) time step, which puts a heavy burden on the CPU and could result in unacceptably long run time. If so, a proper approximate method of taking the integral is required.

The result of integration depends on the specifics of the distribution function, and could vary a lot from one distribution to another. However, for many problems, the distribution function has some a priori known features. For example, let's take it as an even function with a single maximum at zero argument. 
The distribution function of the given type can be characterized by some set of parameters, like its standard deviation (rms value), higher moments, maximal derivative of any order, scalar products with some basis functions, etc. The more parameters - the fuller is the kept knowledge of the function. A general rule for the choice of parameters is trying to minimize their mutual correlations. Let the symbol $\overrightarrow{\boldsymbol{a}}=\left(\boldsymbol{a}_{1}, \boldsymbol{a}_{2}, \ldots.\right)$ mean a list of these parameters of the distribution, or the input function $f(\boldsymbol{v})$ of the transformation Eq. (1).

How can the output of this transformation $\chi(\boldsymbol{u})$ be characterized by its own list of parameters $\vec{A}=\left(A_{1}, A_{2}, \ldots\right)$, so that it could be restored with sufficient accuracy from this list? A possible way to get this output parameterization is the analytical fitting. Namely, some combination of standard mathematical functions $\boldsymbol{F}(\boldsymbol{u}, \overrightarrow{\boldsymbol{A}})$ with unknown coefficients $\overrightarrow{\boldsymbol{A}}$ can be tried to approximate the output function for one or another representative input function. After several cut-and-trials, a reasonable compromise between the accuracy and simplicity of this analytic fitting can be achieved. For every specific input, the coefficients $\boldsymbol{A}$ can be found by minimizing the error $\sum_{i}\left[\boldsymbol{F}\left(\boldsymbol{u}_{i}, \vec{A}\right)-\chi_{i}\right]^{2}$ on some grid $\boldsymbol{u}_{i}$, with $\chi_{i}=\chi\left(\boldsymbol{u}_{i}\right)$. This procedure gives the sought parameterization of the output function, and the mapping $\overrightarrow{\boldsymbol{a}} \rightarrow \overrightarrow{\boldsymbol{A}}$.

The point is that after some preliminary job, the output parameters $\overrightarrow{\boldsymbol{A}}^{\text {new }}$ for any new input function $\boldsymbol{f}^{\text {new }}$ can be found from the input parameters $\overrightarrow{\boldsymbol{a}}^{\text {new }}$ without doing the integral of Eq. (1).

To do that, let some reasonably representative sampling of the input functions $\boldsymbol{f}^{\alpha}$ be taken, and a mapping table $\overrightarrow{\boldsymbol{a}}^{\alpha} \rightarrow \overrightarrow{\boldsymbol{A}}^{\alpha}$ be built. Then, this table can be transformed into a list of functions $\vec{A}(\vec{a}) \equiv\left\{A_{1}\left(a_{1}, a_{2}, \ldots\right), A_{2}\left(a_{1}, a_{2}, \ldots\right), \ldots.\right\}$

by means of any standard numerical interpolation procedure. After that, Eqs. (2) give an alternative procedure for the mapping $\overrightarrow{\boldsymbol{a}} \rightarrow \overrightarrow{\boldsymbol{A}}$, where no integration is required. This way of getting the output is much faster than the direct integration in Eq. (1), while its accuracy can be improved with better choice of the fitting function $\boldsymbol{F}(\boldsymbol{u}, \overrightarrow{\boldsymbol{A}})$, as well as change and increase of the number of the parameters $\overrightarrow{\boldsymbol{a}}$ and $\overrightarrow{\boldsymbol{A}}$.

This procedure of the integral transformation without actual computation of the integral can be called as a "guess method". The method consists of the following steps:

1. Description of the input function $f(v)$ by a list of the input parameters $\overrightarrow{\boldsymbol{a}}$, like moments, max derivatives, etc.

2. Description of the output function $\chi(\boldsymbol{u})$ by its list of parameters. After some way along the learning curve, a good candidate for that description is usually found $\chi(\boldsymbol{u}) \cong \boldsymbol{F}(\boldsymbol{u}, \vec{A})$.

3. Doing calculations for a finite set of the input functions, and getting a mapping table $\overrightarrow{\boldsymbol{a}}^{\alpha} \rightarrow \overrightarrow{\boldsymbol{A}}^{\alpha}$.

4. Interpolation of the table obtained at the previous step by means of a numerical interpolator, and getting the mapping as a list of functions $\vec{A}(\vec{a})$.

5. Using this list of functions whenever the integral transformation (1) is needed, and getting the result as $\chi(u)=F(u, \vec{A}(\vec{a}))$.

The entire procedure $1-5$ is a construction of a "guess machine". It is not fully formalized. Step 1 requires good choice for the input function parameters. Step 2 requires good idea about the output description by means of a combination of standard mathematical functions. However, both not-so-formal steps are usually getting more and more efficient after several cuts and trials. In principle, there are no limits of the guess accuracy: it is increased with better choice and higher number of the input and output parameters.

As soon as the guess machine is constructed, its use makes tremendous saving of the CPU time for the integral transformation, reducing it typically by about two orders of magnitude. 


\section{Example: Dispersion Integral}

A dispersion integral appears whenever the beam response is important. For example, for pure longitudinal dynamics it has a form

$$
\chi(u) \equiv \int \frac{f^{\prime}(v)}{u-v} d v
$$

where $f^{\prime}(v)$ is a derivative of the distribution function, and the principal value of the integral is assumed. An example of a process where these integrals have to be calculated millions of times is stochastic cooling, and this puts a significant burden on the CPU time.

For this example, the dis tribution function $\boldsymbol{f}(\boldsymbol{v})$ is assumed to be a monotonic function of the absolute value of the velocity $|\boldsymbol{v}|$. The following parameters for this function are suggested: rms width $\sigma_{v} \equiv \sqrt{\left\langle v^{2}\right\rangle}$, a maximum of the derivative $f_{\text {max }}^{\prime}$, and the argument where this maximum occurs $\boldsymbol{v}_{\mathbf{0}}$. In fact, the width $\sigma_{v}$ can be treated as a scaling parameter, and be excluded by re-scaling of the argument. After that, only two parameters for the maximum derivative remain as the input function characteristics:

$$
a_{1}=f_{\max }^{\prime} \sigma_{v}^{2}, \quad a_{2}=v_{0} / \sigma_{v} .
$$

After some cut-and-tests, the following combination was accepted for the analytical match of the integral Eq. (3):

$$
\begin{aligned}
& F(u, \vec{A})=\frac{u^{2}-A_{1}^{2}}{u^{4}+A_{1}^{2} / A_{2}}\left[1+\frac{A_{4} A_{5}^{4}}{A_{3}^{2}+A_{5}^{2}} G\right] \\
& G \equiv\left(1-\frac{A_{6}}{u^{2}+A_{7}^{2}}\right) \frac{u^{2}}{\left(u^{2}-A_{3}^{2}\right)^{2}+A_{5}^{4}}
\end{aligned}
$$

At first glance, this formula might seem more complicated than it really is. Indeed, the factor $\boldsymbol{G}$ vanishes both at small and at large $\boldsymbol{u}$; thus, it only corrects results in the intermediate region. So, the factor before the square bracket in $\boldsymbol{F}$ describes the integral in limits of small and large arguments. Taking into account that both $\boldsymbol{F}$ and $\boldsymbol{G}$ must be even functions of $\boldsymbol{u}$, there are not so many relatively simple possibilities for reflection the integral behavior at the limits and for matching in the intermediate range of $\boldsymbol{u}$. Note that Eq. (5) is just a possible analy tical fit of the integral (3), some compromise between the accuracy and complexity of the result.

After the descriptive parameters for the input and output functions are fixed by Eqs. $(4,5)$, it is time to collect "empirical data", getting the specific values of the output coefficients $\boldsymbol{A}$ for various input representatives. These representatives should be constructed as different from each other as possible, i. e. covering as wide as possible area in the space of the input parameters $\boldsymbol{a}$, belonging though to the fixed class of monotone smooth functions. When the table of such data is getting to be as long as $15-20$ rows, the generated data can be considered sufficient, the interpolator applied, and the guess machine is ready. After that, this machine can be tried for a new representative, and if the accuracy is seen as good enough, the guess integrator can be used. Here, I am not going to present the list of my 20 representative functions; instead, a typical example of agreement between the guessed and actual behavior of the integral (4) for some test function is shown in Fig. 1. It is seen that disagreement is maximal at the intermediate range of parameters; the maximal error is $\sim 20 \%$. Any definition of an average error would give not more than a few percent, which was sufficient for my purposes. If needed, the agreement could be improved, introducing more parameters, or modifying the fitting function.

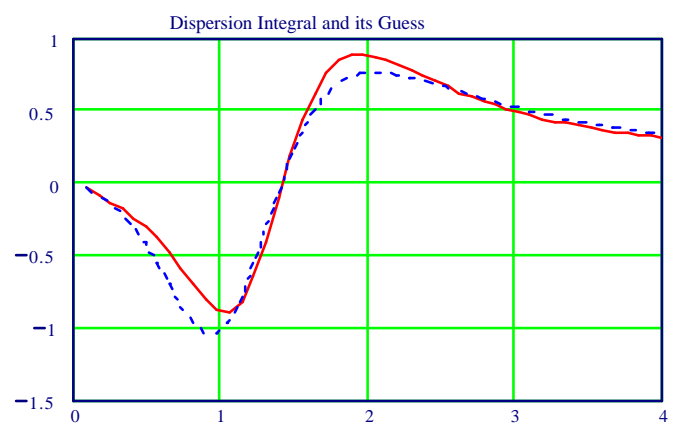

FIGURE 1. Dispersion integral (solid) and its guess (dash) based on 20 representative mappings. This is an example of typical agreement between the two. 


\section{INTEGRALS FOR IBS DIFFUSION AND FRICTION}

Intra-beam scattering (IBS) is described by Landau collision integral [3] which has to be added to the Vlasov equation. Landau collision integral has the Fokker-Planck structure; an evolution of the distribution is described as

$$
\frac{\partial \boldsymbol{f}}{\partial \boldsymbol{t}}-[\boldsymbol{H}, \boldsymbol{f}]=\frac{\partial}{\partial \boldsymbol{p}_{\alpha}}\left(-\boldsymbol{F}_{\alpha} \boldsymbol{f}+\frac{\boldsymbol{D}_{\alpha \beta}}{\boldsymbol{2}} \frac{\partial \boldsymbol{f}}{\partial \boldsymbol{p}_{\beta}}\right),
$$

where the Poisson bracket $[\boldsymbol{H}, \boldsymbol{f}$ ] is the Vlasov part, and the Landau collision term on the right hand side is to be calculated with the friction and diffusion as

$$
\begin{aligned}
& F_{\alpha}(\mathbf{p})=\frac{4 \pi n e^{4} L_{C}}{m} \int f\left(p^{\prime}\right) \frac{u_{\alpha}}{|\mathbf{u}|^{3}} d^{3} p^{\prime} \\
& D_{\alpha \beta}(p)=4 \pi n e^{4} L_{C} \int f\left(p^{\prime}\right) \frac{\mathbf{u}^{2} \delta_{\alpha \beta}-u_{\alpha} u_{\beta}}{|\mathbf{u}|^{3}} d^{3} p^{\prime}
\end{aligned}
$$

where all the symbols are of conventional meaning, and relativism can be easily included if needed.

Scattering term leads to a slow evolution, while the Vlasov term describes fast oscillations. To get rid of this fast motion, a transfer to the action-phase variables and the phase averaging are needed. It leaves only the scattering terms, but both friction and diffusion are expressed as 5D (coasting beam) or 6D (bunched beam) integrals over the evolving distribution. Every such integral is a strongly varying function in the 3D space of actions. Direct computation of these integrals normally takes too long, so approximations are required. Conventionally, the distribution is assumed to be Gaussian for all the 3 degrees of freedom, and growth rates of the beam rms values are calculated, as it was done by Piwinski and Bjorken-Mtingwa. This approach is insufficient when deviations from the Gaussian shape are significant. Often, the distribution is far from Gaussian if cooling is applied. That is why evolution of cooled beams actually requires solution of the kinetic equation. This "detailed" IBS treatment is especially important, when tails of the distribution are of interest, as it is for the losses and lifetime problems. Two cases can be pointed out, when the friction force and diffusion coefficients Eqs. (8) can be reduced to 1D integrals.

\section{Longitudinally Cold Beams}

Let the beam longitudinal temperature be much smaller than the transverse ones. In this case, IBS is essentially reduced to the longitudinal diffusion due to transverse-to-longitudinal heat transfer, and the problem reduces to evolution of the longitudinal distribution. The only integral to be calculated is the longitudinal diffusion coefficient as a function of transverse and longitudinal amplitudes. Let it be assumed that the transverse distribution is Gaussian, and the beam is round transversely. Then, for a bunched beam, the diffusion coefficient is written [4]:

$D \equiv \frac{d}{d t} \mathbf{v}_{z m}^{2}=\frac{4 \pi r_{0}^{2} c L_{C}}{\gamma^{2}}\left\langle\frac{n}{\Delta v_{\perp}}\right\rangle_{x, y, z}=$

$\frac{2 r_{0}^{2} c L_{C}}{\gamma^{2} \sigma_{x}^{2} u_{x}}\left(\frac{v_{x m}}{u_{x}}, \frac{v_{y m}}{u_{x}}\right)\langle\lambda\rangle_{z}$;

$\mathcal{G}(x, y) \equiv \frac{2}{\pi} e^{-\frac{x^{2}+y^{2}}{2}} \int_{0}^{\infty} \int_{0}^{\infty} \frac{I_{0}(\xi x) I_{0}(\eta y)}{\sqrt{\xi^{2}+\eta^{2}}} e^{-\frac{\xi^{2}+\eta^{2}}{2}} d \xi d \eta$

Here $\sigma_{x}$ is the beam transverse rms size, $\boldsymbol{u}_{\boldsymbol{x}}$ is the rms transverse velocity of the beam particles in the beam frame, $\boldsymbol{v}_{\boldsymbol{x} \boldsymbol{m}}, \boldsymbol{v}_{\boldsymbol{y} \boldsymbol{m}}, \boldsymbol{v}_{z \boldsymbol{m}}$ are the velocity amplitudes at the given point of $3 \mathrm{D}$ phase space, and all the velocities are in the units of the speed of light $\boldsymbol{c}$.

With this result, the only value to be integrated is the longitudinal phase average of the bunch linear density

$$
\langle\lambda\rangle_{z} \equiv \int_{0}^{2 \pi} \lambda\left(z\left(\varphi_{z}\right)\right) \frac{d \varphi_{z}}{2 \pi}
$$

The diffusion coefficient of Eq. (9) does not assume anything specific about the longitudinal profile $\lambda(z)$, it can be arbitrary. The only integral transformation which has to be performed every few time steps is $\langle\lambda\rangle_{z}$, Eq. (10). Being 1D, this integral can be taken by the guess method, described above. The IBS diffusion coefficient of Eq. (9) was obtained in Ref [2], where equilibrium was studied between IBS and longitudinal electron cooling of relativistic bunched beams in RHIC. Due to strong dependence of the cooling rate on the particle 3D amplitudes, this dependence has to be kept both in the IBS diffusion and cooling rate.

\section{Stochastic Cooling Against IBS}

Longitudinal stochastic cooling is normally independent of the transverse degree of freedom. That is why the entire 3D distribution function can be considered as factorized in this case, with the Gaussian 
transverse distribution. For this "semi-Gaussian" model, the problem reduces to $1 \mathrm{D}$ longitudinal Fokker-Planck equation. The diffusion coefficient and the friction force of this equation are given by 1D longitudinal integrals. Kernels of these integral transformations are determined by the transverse temperature; the results are presented in Ref. [4]. Note that these 1D integrals can be also taken by the guess method.

\section{ACKNOWLEDGEMENTS}

The author acknowledges many fruitful discussions with Valeri Lebedev.

\section{REFERENCES}

1. A. Burov, "Antiproton Stacking in Recycler", FERMILAB-Conf-03-171, 2003.

2. A. Burov, "Electron Cooling Against IBS for High Energy Colliders", FERMILAB-TM-2213, 2003

3. L. D. Landau, "Kinetic Equation for Coulomb Interaction," Phys. Zs. Sowjet., 10, 154, 1936; also in E. M. Lifshitz and L. P. Pitaevsky, Physical Kinetics, Landau Course of Theoreical Physics, v. 10, Pergamon Press, 1981.

4. P. Zenkevich, O. Boine-Frenkenheim and A. Bolshakov, "Kinetic Effects in Multiple Intra-Beam Scattering", Proc. HB2004, Bensheim, Germany, 2004. 